\title{
Is the methanation reaction over Ru single crystals structure dependent?
}

\author{
Vendelbo, Søren Bastholm; Johansson, Martin; Nielsen, Jane Hvolbæk; Chorkendorff, Ib
}

Published in:

Physical Chemistry Chemical Physics

Link to article, DOI:

10.1039/c0cp02371j

Publication date:

2011

Document Version

Publisher's PDF, also known as Version of record

Link back to DTU Orbit

Citation $(A P A)$ :

Vendelbo, S. B., Johansson, M., Nielsen, J. H., \& Chorkendorff, I. (2011). Is the methanation reaction over Ru single crystals structure dependent? Physical Chemistry Chemical Physics, 13(10), 4486-4493.

https://doi.org/10.1039/c0cp02371j

\section{General rights}

Copyright and moral rights for the publications made accessible in the public portal are retained by the authors and/or other copyright owners and it is a condition of accessing publications that users recognise and abide by the legal requirements associated with these rights.

- Users may download and print one copy of any publication from the public portal for the purpose of private study or research.

- You may not further distribute the material or use it for any profit-making activity or commercial gain

- You may freely distribute the URL identifying the publication in the public portal 
Cite this: Phys. Chem. Chem. Phys., 2011, 13,4486-4493

\title{
Is the methanation reaction over $\mathrm{Ru}$ single crystals structure dependent? $\dagger$
}

\author{
Søren B. Vendelbo, Martin Johansson, Jane H. Nielsen and Ib Chorkendorff \\ Received 2nd November 2010, Accepted 22nd December 2010 \\ DOI: $10.1039 / \mathbf{c 0 c p 0 2 3 7 1 j}$
}

The influence of monoatomic steps and defects on the methanation reaction over ruthenium has been investigated. The experiments are performed on a $\mathrm{Ru}\left(\begin{array}{lll}0 & 1 & 54\end{array}\right)$ ruthenium single crystal, which contains one monoatomic step atom for each 27 terrace atoms. The methanation activity is measured at one bar of hydrogen and $\mathrm{CO}$ in a high pressure cell, which enables simultaneous measurements of the local reactivity of the well defined single crystal surface and the global reactivity of the entire crystal and its auxiliary support. By adding sulfur we observe that the measured activity from the well defined stepped front-side of the crystal is poisoned faster than the entire crystal containing more defects. We also observe that additional sputtering of the well-defined front-side increases the reactivity measured on the surface. Based on this, we conclude that the methanation reaction takes place on undercoordinated sites, such as steps and kinks, and that the methanation reaction is extremely structure dependent. Simulations of the flow, temperature, and product distributions in the high pressure cell are furthermore presented as supplementary information.

\section{Introduction}

The understanding and description of the nature of the active site in heterogeneous catalysis has been an interesting topic for almost a century dating all the way back to the suggestions by Taylor that unsaturated active sites at the atomic level can control the surface chemical reactivity. ${ }^{1}$ The phenomena was discussed in further detail by Boudart who went on to classify reactions in terms of their structure sensitivity or structure insensitivity. ${ }^{2}$ The generality of this approach was later discussed by Yates. ${ }^{3}$ The advent of ultra high vacuum (UHV) technology and surface science methods applied on well defined single crystals has made it possible to establish direct links between the atomic level reactions and heterogeneous catalysis. Such comparisons were pioneered by Goodman and his co-workers for the methanation reaction, $\mathrm{CO}+3 \mathrm{H}_{2} \rightarrow \mathrm{CH}_{4}+\mathrm{H}_{2} \mathrm{O},{ }^{4}$ over nickel and ruthenium. ${ }^{5-7}$ In these studies, single crystals were prepared under UHV and the reactivity was measured at a pressure of nearly one bar. Surfaces such as $\mathrm{Ni}(111), \mathrm{Ni}(110), \mathrm{Ru}(001)$ and $\mathrm{Ru}(110)$ were investigated and a reasonable good correspondence to the activity of heterogeneous catalysts consisting of supported nanoparticles was found. Since no particular differences in reactivity between the different surfaces were identified, it was concluded that the methanation reaction was structure

Center for Individual Nanoparticle Functionality (CINF)

Department of Physics, Technical University of Denmark,

Building 312, $2800 \mathrm{Kgs}$. Lyngby, Denmark

$\dagger$ Electronic supplementary information (ESI) available. See DOI: $10.1039 / \mathrm{c} 0 \mathrm{cp} 02371 \mathrm{j}$ independent. This was puzzling since the methane dissociation, which is the rate limiting step in the reverse reaction (the steam reforming process) was found also by Goodman and coworkers to be structure dependent. ${ }^{8}$ Here, significant differences between the dissociation probability of methane on $\mathrm{Ni}(111), \mathrm{Ni}(100)$, and $\mathrm{Ni}(110)$ were found. However, within the last decade it has been realized that even small amounts of defects may actually completely dominate the reactivity of such planar surfaces. For instance, it was shown by combining surface science work and density functional theory (DFT) calculations that mono-atomic steps were at least nine orders of magnitude more reactive than the terrace sites for the dissociation of nitrogen on ruthenium. This dramatic effect was found to originate from a combination of an electronic effect due to the change in the electronic structure of the undercoordinated step atom and a geometric effect from having a reaction site offering five $\mathrm{Ru}$ atoms instead of only four on the terrace. ${ }^{9}$ With such differences in activity, the small amounts of steps and defects that are always present on even the best single crystal surfaces may completely dominate the reactivity and render more subtle differences e.g. between $\mathrm{Ni}(111)$ and $\mathrm{Ni}(110)$ surfaces completely insignificant. Subsequently, the structure dependence has been investigated for both the steam reforming process and the methanation reaction ${ }^{10-12}$ and in both cases the structural dependence is very clear. The rate limiting step for the methanation reaction is generally believed to involve the dissociation of $\mathrm{CO}^{11,13,14}$ Recently, it was shown that on nickel under UHV conditions, the reaction pathway involves $\mathrm{CO}$ dissociation while at high pressure and in the presence of hydrogen, a $\mathrm{COH}$ intermediate 
is the precursor for dissociation. ${ }^{11}$ The latter was deduced from measurements on catalysts combined with DFT calculations. There is therefore a need to further confirm, by combining the detailed insight single crystal experiments provide with high pressure experiments as performed initially by Goodman and co-workers ${ }^{5}$ that the steps are also dominating under conditions where the $\mathrm{COH}$ reaction pathway may prevail.

The methanation reaction is a technologically important reaction used in industry to remove $\mathrm{CO}$ and $\mathrm{CO}_{2}$ from the hydrogen supply for ammonia production. ${ }^{15,16}$ The process has also recently gained much interest since it is proposed to replace the preferential oxidation of $\mathrm{CO}(\mathrm{PROX})^{17}$ for removing similar remote amount of $\mathrm{CO}$ from the hydrogen feed to Proton Exchange Membrane Fuel Cells. ${ }^{18}$ Methanation is also used in connection with gasification of coal, where methane is produced from synthesis gas ${ }^{19}$ and in relation to Fischer-Tropsch synthesis. ${ }^{20}$ Nickel is used as the preferred methanation catalyst due to its selectivity towards methane and its favorable price, but the reactivity of ruthenium surpasses that of nickel. ${ }^{21-23}$ Ruthenium may therefore be used where the advantage of the higher reactivity and/or resistance towards carbon deposition overrules the disadvantage of the price.

In this work, the methanation reaction over ruthenium has been studied to gain fundamental insight into the reaction mechanism and the active site of the methanation reaction. Yates and co-workers have previously used isotopic scrambling and infrared spectroscopy of labeled $\mathrm{CO}$ to demonstrate that $\mathrm{CO}$ dissociates at the steps on ruthenium, ${ }^{24,25}$ and recent data in our group confirm this result. ${ }^{26}$ We have chosen to work on a stepped single crystal, and to either decrease the number of available steps by adsorbate blocking or to increase the step density by argon sputtering. Different adatoms can be chosen in a step-blocking experiment. We have chosen to use sulfur which is a well-known inhibitor or poison to active sites in general $^{23,27,28}$ due to its strong interaction with undercoordinated sites. ${ }^{29,30}$ In particular for the methanation reaction over ruthenium, Goodman and co-workers have shown that less than $5 \%$ of a monolayer of sulfur was needed to poison up to $70 \%$ of the methanation activity on a $\mathrm{Ru}(001)$ crystal, see ref. 31-33 and references therein. This effect was explained by a change in the electronic structure of up to ten surrounding ruthenium atoms, which was a reasonable explanation at that time. More recently, however, it has become clear that such interactions will be screened out at longer distances in metallic systems, and probably only have an influence on nearestneighbors (see ref. 15 and references therein). The stepblocking experiment requires an adsorbate which sticks to the steps even under reaction conditions at high temperatures and pressures. In the study of nitrogen dissociation on ruthenium we used gold to block the ruthenium steps, ${ }^{9}$ but we have strong evidence that $\mathrm{CO}$ can push the gold atom away from the step due to the strong interaction of the $\mathrm{CO}$ with the step at high pressures. Such an effect has been observed by STM in the Besenbacher group for gold on Ni(111), ${ }^{34}$ and we therefore refrain from using gold and use sulfur as our stepblocking agent instead.

With the purpose of increasing the number of step sites we sputter-damage our surface. One could also have chosen a single crystal with a larger miscut angle compared to the low index surface normal. In that case, however, care should be taken because highly stepped single crystals may not be stable at the temperatures and/or pressures used for these reactivity studies, since gas can introduce reconstructions and redistribution of material on the surface. ${ }^{35-37}$

When performing reactivity measurements it is very important to be able to control where the reaction products come from. In the following we shall distinguish between a global and a local measurement: In a global measurement all the reaction products originating from anywhere in the reaction cell are measured. Here it is not possible to know if the products come from the front-side surface under investigation, from the rim or backside of the sample, from the heating system (e.g. hot filaments), from the thermocouple attached to the crystal, or even from the walls of the reaction cell. For example, Madey and co-workers have shown that there is a significant activity measured from a tungsten surface. ${ }^{38}$

The local measurement is performed by positioning a capillary connected to a quadrupole mass spectrometer (QMS) very close to the single crystal surface. More details will be given below.

The work presented in this paper is the first to measure the difference between the methanation activity on ruthenium terraces and surface defects such as steps and kinks at pressures up to one bar. This is done by comparing global and local measurements of the reactivity, which allows us to conclude on the active site for the methanation reaction, and to measure the effect of decreasing and increasing the number of defect sites using sulfur and sputtering, respectively. The interpretation is furthermore supported by computational fluid dynamics (CFD) calculations (see Supplementary material) confirming that an adequate separation of the signals is possible and that the assumed pressures and temperature gradients are valid.

\section{Experimental}

All the experiments are conducted in a stainless steel UHV chamber. Auger electron spectroscopy (AES) is used to characterize the surface composition using a Perkin Elmer cylindrical mirror analyzer. The sample is a $\operatorname{Ru}\left(\begin{array}{lll}0 & 1 & 54\end{array}\right)$ disc shaped single crystal supplied by Mateck, with a diameter of $10 \mathrm{~mm}$ and a height of $4 \mathrm{~mm}$ with only the front surface polished. Consequently, it has an average step density of $4 \%$ with terrace widths of approximately 27 atoms. The rest of the crystal is not polished. The sample was mounted with a tungsten filament which was used for direct current heating. A C-type (tungsten, 5\% rhenium and tungsten, 26\% rhenium) thermocouple was spot welded on to the side of the single crystal. This type of thermocouple does not contain Ni which could form nickel carbonyls in the presence of $\mathrm{CO}$ gas, ${ }^{39}$ resulting in nickel contamination of the ruthenium surface.

Initially, the ruthenium crystal was cleaned by extensive sputtering and annealing cycles in oxygen to remove carbon. ${ }^{26}$ The crystal was cleaned before reactivity measurements in the following way: First, an oxidation step at $1450 \mathrm{~K}$ in $10^{-7}$ torr oxygen for 2 min was performed to remove sulfur residues. Then, the surface was sputtered with $1 \mathrm{keV}$ argon ions at 
$800 \mathrm{~K}$ for $30 \mathrm{~min}$. The sample was then heated in $10^{-7}$ torr oxygen to $1100 \mathrm{~K}$ for $10 \mathrm{~min}$ to remove carbon which is the primary bulk contaminant. After this, any remaining oxygen on the surface was removed by dosing hydrogen for $30 \mathrm{~min}$ at a pressure of $10^{-6}$ torr. Finally, the sample was annealed for $1 \mathrm{~min}$ to $1200 \mathrm{~K}$ to remove any oxygen residues. This step furthermore anneals out any remaining defects generated by the sputtering. In order to check that this cleaning procedure did not result in any remaining carbon, an oxygen titration experiment (not part of the standard cleaning procedure) was performed, and no carbon residues were detectable. The titration method was previously demonstrated on the same setup to have a carbon sensitivity below $0.001 \mathrm{ML}^{39}$

Following the cleaning procedure, a temperature programmed desorption (TPD) experiment with $\mathrm{CO}$ was performed. The TPD curve shape is very sensitive to how well-ordered the surface is. Our CO TPD curves resemble previously published curves from well ordered $\mathrm{Ru}(001)$ surfaces. ${ }^{25,40}$ After this cleaning procedure the structure of the surface e.g. the average terrace width remains the same according to scanning tunneling microscopy performed on a similar crystal. ${ }^{41}$

A high pressure cell made from nickel-free steel was connected to the UHV chamber, see Fig. 1, and two quadrupole mass spectrometers were used, a Baltzers 125 and a Baltzers 400, ensuring simultaneous measurements of the local and the global reactivities. Pressures up to one bar could be reached. The pressure is limited only by the bellows and the
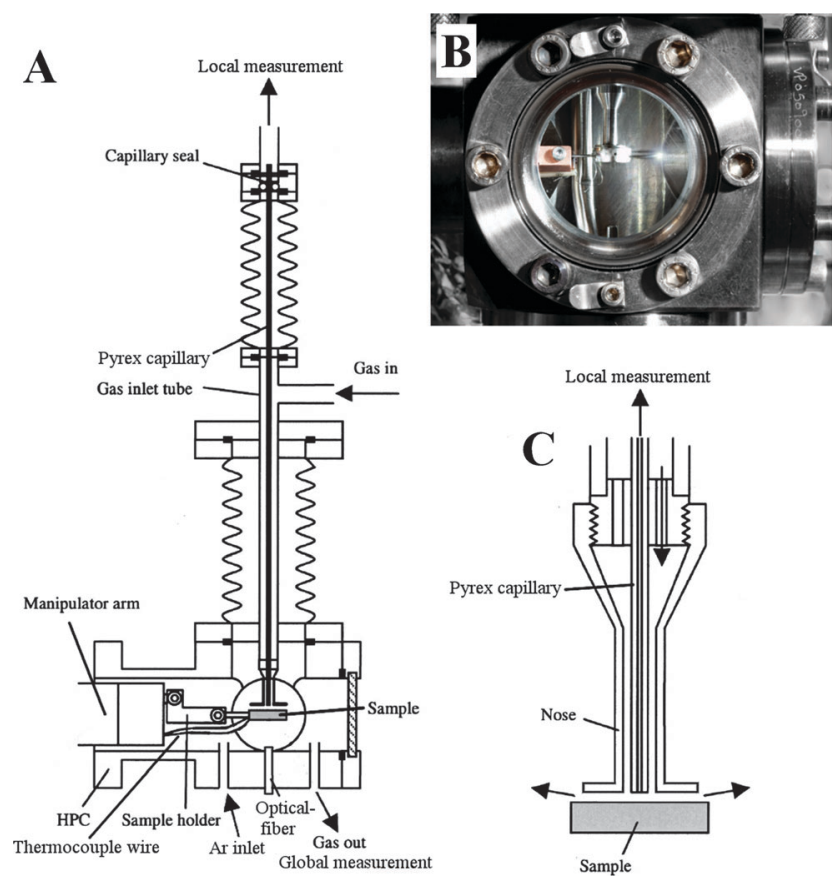

Fig. 1 The HPC and gas inlet shown schematically (A). The arrows indicate the direction of the gas flow. A photograph of the HPC is shown in (B) where the nozzle is seen in close proximity with the single crystal surface which is mounted on tungsten wires connected to copper pins. A cross section of the nozzle close to the sample is seen in (C). The local measurement is performed just below the nozzle at the sample surface, and the global measurement is performed at the outlet of the HPC situated in the lower part of (B). The three images are not shown on the same scale. windows of the HPC. In the HPC, an aluminium nozzle is positioned right above the sample with its long axis normal to the surface. Aluminium with a purity better than $99.999 \%$ was used since it was previously found that aluminium alloys could be a source of sulfur and $\mathrm{Mg}$ when heated during experiments. The aluminium nozzle can be positioned relative to the ruthenium surface by a linear motion. The reaction gas is let into the HPC through the nozzle. Inside the nozzle, a Pyrex capillary is mounted concentrically. An additional bellow allows the capillary to move relative to the position of the nozzle. The gas outlet is at the bottom on the HPC together with an argon inlet and an optical feedthrough. When the nozzle is close to the sample and the inlet flow is high, the flow below the nozzle will separate the detection volume of the capillary from the surroundings by creating a diffusion barrier. This is described in more detail in the supplementary material. The result is that the QMS connected to the capillary primarily measures locally on the well-defined front-side of the crystal whereas the second QMS measures on the gas outlet, corresponding to a global measurement as illustrated in Fig. 1. Previously, this method has been employed in the study of the reactivity of alloy spots supported on a graphite surface, ${ }^{42,43}$ but it has not been used for metallic single crystals before.

All reaction gases used on the chamber were of high purity (99.995\% or better). Before entering the HPC, the CO gas was cleaned additionally by using an activated carbon trap, then a cooling trap to condense nickel carbonyls and hydrocarbons, and finally a Pall Mini-Gaskleen purifier which removes any additional nickel carbonyls. Nickel carbonyls may be formed when having high pressures of $\mathrm{CO}$ in stainless steel tubes and result in very reactive adatom adsorption and hence faulty enhancement of the apparent surface reactivity. ${ }^{39,44}$ When using the additional cleaning precautions described above we did not detect any nickel with AES after a methanation experiment. All tubes containing high pressures of $\mathrm{CO}$ following the traps, were made from copper. The hydrogen for the HPC was cleaned using an activated copper catalyst.

Methanation was performed at a pressure of 1 bar in a flow

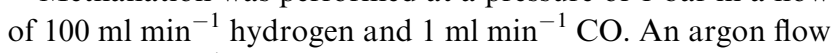
of $1.6 \mathrm{ml} \mathrm{min}{ }^{-1}$ was let directly into the HPC, bypassing the inlet nozzle. This was done to establish an appropriate background measurement for each of the local reactivity measurements (see supplementary material). The temperature was varied between $500 \mathrm{~K}$ and $700 \mathrm{~K}$ in intervals of $25 \mathrm{~K}$. In order to improve the signal-to-noise ratio of the methane signal, the mass-to-charge ratio of 15 was monitored in the mass spectrometer and will be reported in the following (the mass-to-charge ratio of 16 may have contributions from e.g. $\mathrm{O}_{2}, \mathrm{CO}$, and $\mathrm{H}_{2} \mathrm{O}$ ).

The mass spectrometer signal for a mass-to-charge ratio of $x$ denoted $I_{x}$ depends on the gas density, and therefore on the absolute gas temperature which is identical to the temperature at the tip of the capillary, $T$, and the partial pressure of the gas, $\mathrm{p}_{x}$, according to this equation:

$$
I_{x}=s_{x} \frac{p_{x}}{T^{a}}
$$


where $s_{x}$ is a sensitivity factor and $a$ is close to $1 .{ }^{45}$ For simplicity, it is assumed that only one gas is contributing to $I_{x}$. By normalizing the mass spectrometer signals to the one obtained from hydrogen, which has a known, near constant partial pressure, it is possible to remove the temperature dependence. Hence,

$$
p_{x}=p_{H_{2}} \frac{I_{x}}{I_{H_{2}}} \frac{s_{H_{2}}}{s_{x}}
$$

The methane signal was calibrated against a known partial pressure of methane, and the amount of CO consumed was found to be equal to the amount of methane produced.

After a methanation experiment, the amount of carbon deposited during the reaction was determined using oxygen titration. While keeping the sample at $700 \mathrm{~K}$, oxygen to a pressure of $1.5 \times 10^{-7}$ torr was introduced into the chamber and the resulting $\mathrm{CO}$ desorbing from the surface was quantified with the QMS. As mentioned previously, this titration method has a carbon sensitivity down to $0.001 \mathrm{ML}^{39}$

Sulfur was deposited on the ruthenium surface from doses of hydrogen sulfide at a pressure of $5 \times 10^{-8}$ torr while keeping the surface at $600 \mathrm{~K}$. To ensure a uniform sulfur coverage on both sides, the sample was turned halfway through the dosing period to avoid a higher concentration on the surface facing the doser. The gas system for handling the $\mathrm{H}_{2} \mathrm{~S}$ gas was totally disconnected from the regular gas handling system to avoid sulfur contamination of the high purity reaction gasses. The amount of sulfur on the sample was quantified with AES and coverages down to 0.04 ML were detectable $(1 \mathrm{ML}$ is defined as the $\mathrm{Ru}(001)$ surface density of $1.58 \times 10^{19} \mathrm{~m}^{-2}$ ). This rather high detection limit is due to the energy overlap of AES peaks for sulfur and ruthenium. The sulfur coverage, however, was linearly proportional to the dosing time for the short exposures, so for sulfur depositions aiming at coverages below $0.1 \mathrm{Ru} \mathrm{ML}$, only the dosing time was used for quantification. The saturation coverage of sulfur on $\mathrm{Ru}(001)$ is $0.5 \mathrm{ML}^{46}$

The heating filaments used on the setup were made of tungsten, and since tungsten is known to act as a methanation catalyst, ${ }^{38}$ it was important to check that the contribution to the measured activity from these filaments was negligible. For this purpose, an infrared laser was used to heat the backside of the crystal instead of running current through the filaments. The laser was an Amtron LS453 laser with a wavelength of $940 \mathrm{~nm}$. The crystal was held at a temperature of $520 \mathrm{~K}$ using a laser power of $22 \mathrm{~W}$, and both the local and global methane signals were now monitored while switching between laser heating and filament heating, keeping the sample temperature constant. No considerable changes to the local or global methane signals were observed, and we therefore conclude that the filaments or other hot spots in the vicinity do not contribute significantly to either the global or local reactivity measurements.

\section{Results}

In Fig. 2, the raw global (Fig. 2A) and local (Fig. 2B) QMS signals for hydrogen (2 AMU), $\mathrm{CO}$ (28 AMU), and $\mathrm{CH}_{4}$ (15 AMU) are shown. The signals are shown as a function of time, while the temperature as shown in Fig. $2 \mathrm{C}$ is first increased to $700 \mathrm{~K}$ and subsequently decreased to $400 \mathrm{~K}$ in steps of $25 \mathrm{~K}$ or $50 \mathrm{~K}$. At $700 \mathrm{~K}$, a clear methane production is seen which decreases with decreasing temperature. Several masses in addition to the ones shown in Fig. 2 were measured and at no time did we detect higher masses corresponding to longer hydrocarbon chains. Along with the increase in the methane signal, the $\mathrm{CO}$ and hydrogen signals decrease. The majority of this drop is, however, not due to the consumption of the gas (even at full conversion, only $3 \%$ of the hydrogen signal should disappear and this would hardly be visible in the figure) but merely due to a local heating of the gas with a resulting decrease in density, see eqn (1).

The amount of carbon on the surface after methanation is expected to be very low due to the surplus of hydrogen which will react off any remaining carbon and form methane. The lack of carbon is verified by performing an oxygen titration experiment, and the carbon coverage after methanation was found to be less than $0.01 \mathrm{ML}$.

\section{Activation energy}

The global and the local methane signals are proportional to the methane concentrations, which again are proportional to the methane formation rate. It is therefore possible to construct an Arrhenius type plot of the methane concentration as a function of the temperature and thereby extract apparent activation energies.

The global signal is directly converted to a concentration using the ideal gas law, but unintended contributions to the local signal from the global reactor (see further discussions of this in the Supplementary material) must be subtracted before converting to a local concentration. The global contribution to a local signal is determined in each experiment by backfilling the HPC with argon and measuring the resulting local argon signal. The ratio between the local and the global argon signals are now used to scale the global methane signal and subtract this contribution to the local signal.

The average of six measurements of the methane concentration is plotted in Fig. 3 as a function of the inverse sample temperature. The blue points are the global measurements and the red points are the local. Both sets of data are seen to show Arrhenius behavior in parts of the temperature range. The error bars shown are the standard deviation on the average of the concentration values for each temperature. Above $650 \mathrm{~K}$, the global signal levels out as it approaches full conversion, whereas the local methane concentration continues to increase up to the maximum temperature of $700 \mathrm{~K}$. At temperatures below $550 \mathrm{~K}$, the local measurements level off as it reaches the noise level of the QMS. The apparent activation energies can be extracted by fitting the Arrhenius equation to the data. The global activation energy was found to be $0.83 \pm 0.02 \mathrm{eV}$ fitted from $500 \mathrm{~K}-625 \mathrm{~K}$, whereas the local activation energy was found to be $0.72 \pm 0.10 \mathrm{eV}$ fitted from $550 \mathrm{~K}-700 \mathrm{~K}$. The fitting intervals are indicated with straight lines in the figure. The error bar on the activation energy is based on the error bars of each data point. The relative standard deviation on the global activation energy is much smaller than for the local 


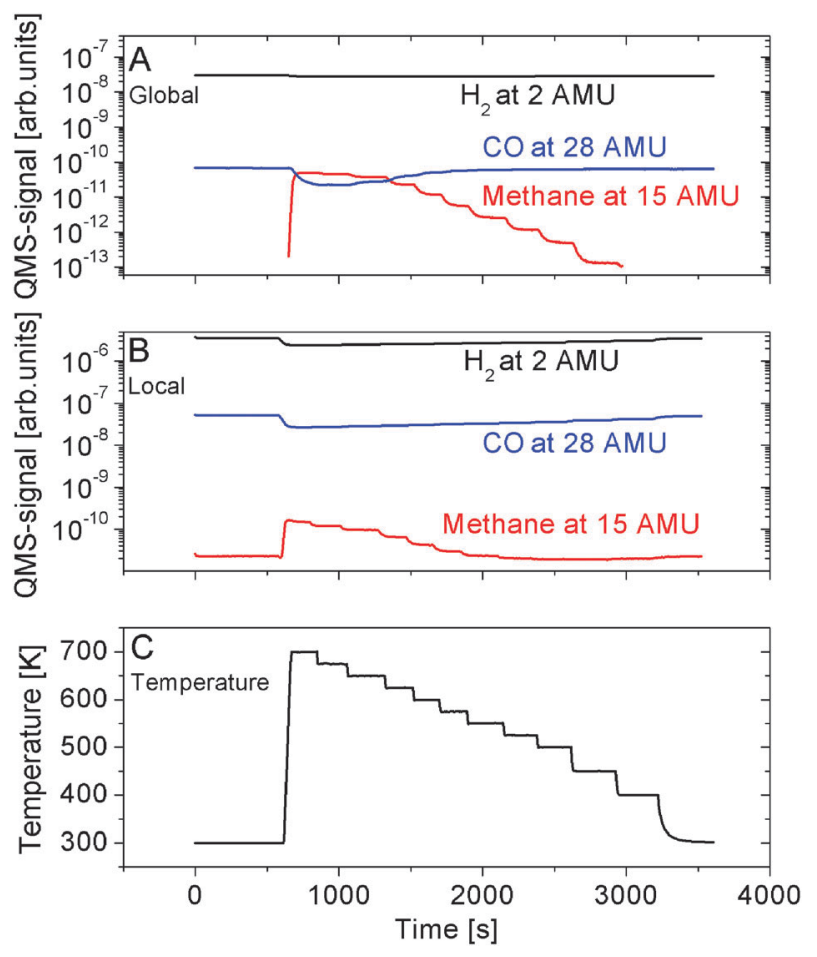

Fig. 2 The measured global (A) and local (B) QMS signals are shown as a function of time. At $t=600$, the temperature (C) is increased to $700 \mathrm{~K}$, and decreased in steps down to $400 \mathrm{~K}$. The hydrogen signals (mass 2) are black, the $\mathrm{CO}$ signals (mass 28) are blue, and the methane signals (mass 15) are red.

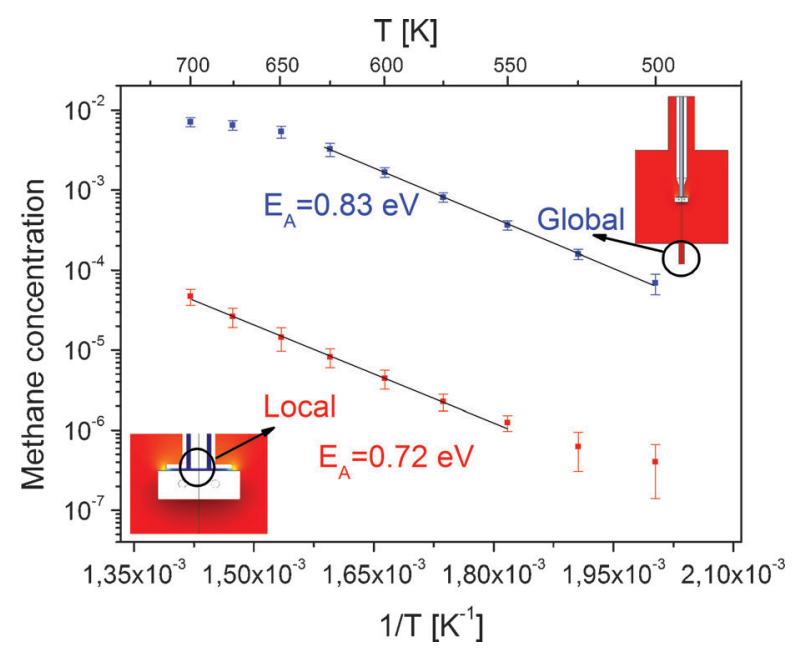

Fig. 3 An Arrhenius plot of the methane molar fraction which is proportional to the rate (see text). The blue points are the global measurements and the red points are the local measurements. The activation energies are the average apparent activation energy from many experiments. The lines indicate in what temperature interval the activation energies are calculated. The error bars are the standard deviation of six different experiments. The insets show where in the reactor the concentration was measured.

activation energy because the local signal is much smaller on an absolute scale than the global one.

The TOF calculated from eqn (5) in the supplementary material, using the global concentration (proportional to the rate) assuming a ruthenium surface density of $1.58 \times 10^{19}$ atoms $^{-2}$ is found to be between 68 and 0.6 molecules/atom/s in the temperature range from $700 \mathrm{~K}$ to $500 \mathrm{~K}$.

\section{Sulfur poisoning}

In order to block surface defects, hydrogen disulfide is dosed resulting in adsorbed sulfur, initially at the step sites. Measurements of the methanation reaction rate at $550 \mathrm{~K}$ are then carried out in the HPC as a function of sulfur coverage, see Fig. 4. Both the local and the global signals decrease upon addition of even small amounts of sulfur. The local methane activity decreases by close to $50 \%$ at a sulfur coverage of 0.01-0.02 ML, after which it decreases more slowly to $25 \%$ of the initial value at $\sim 0.10 \mathrm{ML}$ sulfur. The global reactivity decreases slower by $\sim 10 \%$ after adsorption of $\sim 0.01-0.02 \mathrm{ML}$, and reaches half its original value after adsorption of $\sim 0.1$ ML. At a sulfur coverage close to saturation at $0.5 \mathrm{ML}$, both the local and global signals are less than $5 \%$ of the initial value of the clean ruthenium surface. At zero sulfur coverage, the average of six measurements is plotted along with the standard deviation. Since the methanation reaction of a clean surface is extremely sensitive to the defect level and in particular to the cleanliness, even remote amounts of impurities or changes in preparation of the surface may lead to fluctuations, reflected in the rather large error bar on the reactivity of the clean crystal. This error bar is only used at the clean surface since small sulfur residues on a crystal which already contains sulfur are not significant.

\section{Effect of sputtering}

As previously mentioned, adding more defects to the surface can also give valuable information about the active site. The cleaned ruthenium surface was therefore sputtered, after cleaning, at $300 \mathrm{~K}$ for $30 \mathrm{~min}$ with $1 \mathrm{keV} \mathrm{Ar}^{+}$ions, and the methanation rate was then measured in the HPC. In Fig. 5, both the local and the global methane concentrations (rates) for the sputtered ruthenium surface are shown along with results for the clean surface and the sulfur covered one ( $\sim 0.05 \mathrm{ML})$ from Fig. 4 . It is seen that the local activity of the

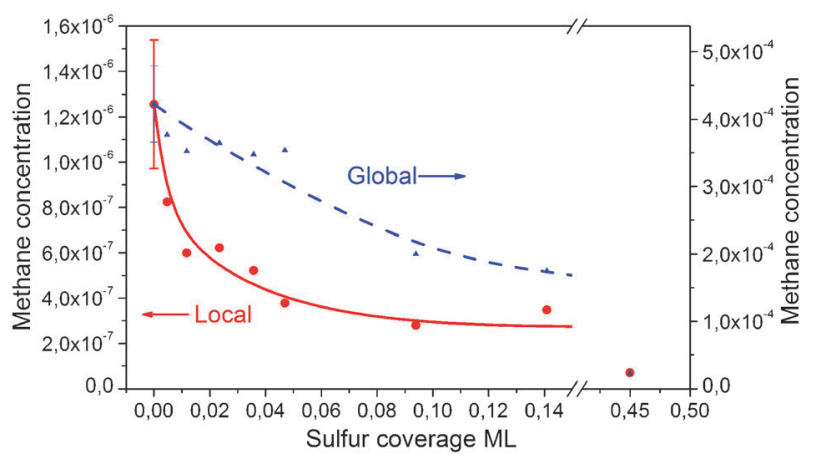

Fig. 4 The methane molar fraction at $550 \mathrm{~K}$ normalized with the hydrogen signal as a function of sulfur coverage. The blue points are the global measurements; the red points are the local. The points at zero sulfur coverage are averages of six measurements. The error bar is the standard deviation of these six measurements. The lines are just to guide the eye. Notice the break in the sulfur coverage axis between $0.15 \mathrm{ML}$ and $0.4 \mathrm{ML}$. 


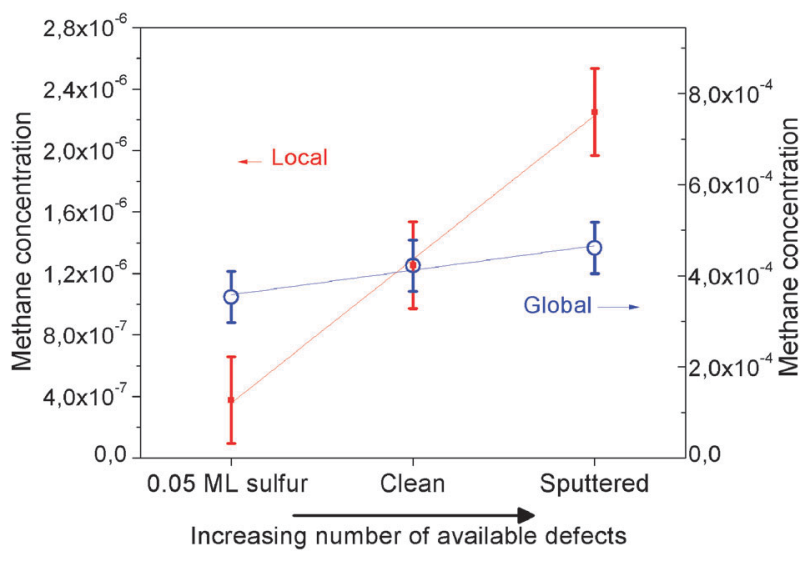

Fig. 5 The methane concentration (rate) plotted for the sulfur covered, clean, and sputtered surfaces. The defect density is increasing towards the right. The red squares are the local measurements and the blue circles are the global measurements. The error bars are the standard deviation on the average of six measurements. Increasing the defect density increases the methanation rate. The lines are merely guides to the eye.

sputtered surface is twice that of the cleaned and annealed surface. There are indications as measured by TPD that the sputtering leads to more roughening, but the subsequent treatment in the high pressure cell seems to anneal the surface so that only a factor of two is observed. The additional drop by a factor of three when adding $\sim 0.05 \mathrm{ML}$ sulfur as reported in Fig. 5 is also seen. The corresponding variation in the global rate follows the same trend, even though the variation is within the error bar of the measurements.

\section{Discussion}

According to Fig. 3, concentration (rate) measurements show Arrhenius behavior over a $150 \mathrm{~K}$ wide temperature range. At temperatures above $650 \mathrm{~K}$, the system was close to full conversion and the global rate levels out. The locally measured rate did not level out at this temperature, so this effect can be attributed to mass transfer limitations due to the consumption of the $\mathrm{CO}$ and not to changes of the ruthenium catalyst. Goodman and coworkers concluded that their methanation rate measured at a CO-to-hydrogen ratio of $1 / 4$ leveled out due to carbon formation on the surface (at 10 torr this happened around $600 \mathrm{~K}){ }^{5} \mathrm{We}$ did, however, not observe significant carbon formation (the carbon formation was below $0.01 \mathrm{ML}$ according to our oxygen titration experiments), and we attribute this to our much smaller CO-to-hydrogen ratio of $1 / 100$. The fact that we also see a higher average TOF than Goodman and co-workers $\left(0.002-10 \mathrm{~s}^{-1}\right.$ in the same temperature range as our values of $0.6-68 \mathrm{~s}^{-1}$ ) can have two explanations: First of all we do not as mentioned earlier expect to see much inhibition since our $\mathrm{CO}$ pressure is substantially lower than that used by Goodman and co-workers. Secondly, our crystal is more defected than the one used by Goodman, i.e. $\mathrm{Ru}(001)$, and since such sites are more reactive, we should expect higher TOF values.

The apparent activation energy for the global experiments of the methanation reaction found using the global signal to be
$0.83 \pm 0.02 \mathrm{eV}$ is lower than the $1.2 \mathrm{eV}$ found by Kelley and Goodman on $\mathrm{Ru}(110)$ and $\mathrm{Ru}(001) .^{5}$ This can be rationalized by their higher $\mathrm{CO}$-to-hydrogen ratio, causing more $\mathrm{CO}$ to be present which can block reaction sites, and thus increase the apparent activation energy.

Measurements of the methanation reaction on ruthenium nanoparticles in the literature result in apparent activation energies from $0.8-1.2 \mathrm{eV}$ depending on the gas compositions used. $^{21,47,48}$ In comparison, our data lies in the lower end of this range- however in agreement with these results, indicating that we probe the same type of active site as in the supported catalyst experiments. For the local measurements, the apparent activation energy was found to be $0.72 \pm 0.10 \mathrm{eV}$. The activation energy is again in agreement with the methanation literature mentioned above though still in the lower end of the energy range. The relative error is seen to be larger for the local compared to the global results. This is due to the fact that the local methane signal is much smaller.

It is interesting to note that our activation energies are substantially smaller than the one found for $\mathrm{CO}$ dissociation under UHV conditions. ${ }^{26}$ There, the experimental values were $1.3-1.5 \mathrm{eV}$ while the theory predicted $1.4 \mathrm{eV}$. The much lower values consistently found for the methanation reaction is ascribed to $\mathrm{COH}$ being an important intermediate for which the barrier is substantially lower which we have previously found to be the case for methanation on nickel. ${ }^{11}$

Sulfur poisoning of the methanation reaction was also previously studied by Goodman and co-workers. ${ }^{31}$ They observed a rapid decrease in the methanation TOF with sulfur coverage. They suggested that the strong effect of sulfur was due to an electronic effect induced by the sulfur atom on the surrounding ruthenium atoms. In Fig. 4, we report a similar strong effect of sulfur poisoning in agreement with Goodman and coworkers, and here our specialized setup enabling both a local and a global measurement reveal new insight: It is seen that the well-ordered surface of the crystal probed by the local measurement is severely affected by sulfur poisoning, decreasing the local reactivity to half its initial value with only 0.01-0.02 ML sulfur added to the surface. At the same time, the global measurement which primarily measures the reactivity of other rougher surfaces drops by only $10 \%$. In a $\mathrm{CO}$ desorption experiment performed on a surface with either $0.04 \mathrm{ML}$ sulfur or $0.04 \mathrm{ML}$ carbon preadsorbed, the desorption peak attributed to dissociated $\mathrm{CO}$ is not present, indicating that the step sites are blocked by either sulfur or carbon. ${ }^{25,26}$ If the sulfur poisoning is due to an electronic effect, a rough and a smooth surface should be poisoned equally fast, and hence the global and the local reactivity behavior when adding sulfur should be the same. This is not the case as seen in Fig. 4 so we attribute the difference between the local and global sulfur poisoning behaviors to be due to the fact that methanation takes place at surface defects such as kinks and steps. Only small coverages of sulfur are needed to poison the steps on the well-defined front-side of the crystal, whereas the rough parts of the crystal need more sulfur to be passivated.

When the well-defined front-side of the crystal was sputtered the local methane signal increased by a factor of two, see Fig. 5. This supports our interpretation since the 
number of defects increase. The defect density is probably increased more than a factor of two when sputtered, but when the crystal is heated at high pressure during the methanation reaction, many of these defects are expected to anneal out. The increase in the global methane signal after sputtering the frontside is only $10 \%$. Since the area of the well-defined front-side of the crystal corresponds to $28 \%$ of the entire area, this reflects the difference in the defect density between the welldefined front-side and the rest of the crystal. So the global signal does not increase much because the reactivity of the well-defined front-side surface only contributes with a small amount to the global measurement. This illustrates the importance of performing a local measurement.

These experiments confirm the strong impact the number of surface defects has on the methanation rate - and also that highly specialized measurements with a high local sensitivity are needed to reveal this.

\section{Conclusion}

We have performed experiments in a specially designed HPC which enables simultaneous measurements of the reactivity at a local spot on the crystal and of the entire crystal and the surroundings.

- It is clearly demonstrated that sulfur has a strong poisoning effect on the well defined surface. The drop in activity correlates with the defect density. The effect of sulfur on the global signal is much less pronounced.

- It is clearly demonstrated that increasing the amount of defects with sputtering increases the reactivity of the surface.

- Despite the fact that it is an experimental challenge to perform rate measurements on single crystals and in particular on species that constitutes a few percent of the surfaces, we are of the opinion that we here have demonstrated that the methanation reaction is extremely structure dependent. This suggests that whenever calculations and fundamental surface science experiments are performed on well defined systems the question should be posed: What is the role of the step and defect sites? They may not always be of importance since their higher reactivity may lead to blocking during the high pressure reaction. Nevertheless, the present study presents an example where such step and defect sites are of imperative importance for understanding the reaction pathway and the rate limiting step of the methanation reaction.

\section{Acknowledgements}

CINF is funded by the Danish National Research Foundation.

\section{References}

$1 \mathrm{H}$. S. Taylor, A theory of the catalytic surface, Proceedings of the Royal Society of London Series A-Containing papers of a mathematical and physical character, 1925, 108(745), 105-111.

2 M. Boudart, Adv. Catal., 1969, 20, 153.

3 J. T. Yates, Vacuum, 1981, 31, 715-722.

4 P. Sabatier and J.-B. Senderens, New methane synthesis, Comptes Rendus Hebdomadaires des Seances de l'academie des Sciences, 1902, 134, 514-516.

5 R. D. Kelley and D. W. Goodman, Surf. Sci., 1982, 123(2-3), L743-L749.
6 D. W. Goodman, R. D. Kelley, T. E. Madey and J. T. Yates, J. Catal., 1980, 63(1), 226-234.

7 D. W. Goodman and J. M. White, Surf. Sci., 1979, 90(1), 201-203.

8 T. P. Beebe, D. W. Goodman, B. D. Kay and J. T. Yates, J. Chem. Phys., 1987, 87(4), 2305-2315.

9 S. Dahl, A. Logadottir, R. C. Egeberg, J. H. Larsen, I. Chorkendorff, E. Törnqvist and J. K. Nørskov, Phys. Rev. Lett., 1999, 83(9), 1814-1817.

10 G. Jones, J. G. Jakobsen, S. Shim, J. Kleis, M. P. Andersson, J. Rossmeisl, F. Abild-Pedersen, T. Bligaard, S. Helveg, B. Hinnemann, J. R. Rostrup-Nielsen, I. Chorkendorff, J. Sehested and J. K. Nørskov, J. Catal., 2008, 259(1), 147-160.

11 M. P. Andersson, E. Abild-Pedersen, I. N. Remediakis, T. Bligaard, G. Jones, J. Engbæk, O. Lytken, S. Horch, J. H. Nielsen, J. Sehested, J. R. Rostrup-Nielsen, J. K. Nørskov and I. Chorkendorff, J. Catal., 2008, 255(1), 6-19.

12 J. K. Nørskov, T. Bligaard, B. Hvolbæk, F. Abild-Pedersen, I. Chorkendorff and C. H. Christensen, Chem. Soc. Rev., 2008, 37(10), 2163-2171.

13 J. W. E. Coenen, P. F. M. T. Vannisselrooy, M. H. J. M. Decroon, P. F. H. A. Vandooren and R. Z. C. Vanmeerten, Appl. Catal., 1986, 25(1-2), 1-8.

14 S. Shetty, A. P. J. Jansen and R. A. van Santen, J. Phys. Chem. C, 2008, 112(36), 14027-14033.

15 I. Chorkendorff and J. W. Niemantsverdriet, Concepts of Modern Catalysis and Kinetics, Wiley-VCH, 2003, ISBN 3-527-30574-2.

16 Haldor Topsoe A/S. Low temperature methanation catalyst PK-7R. http://www.topsoe.com/business_areas/ammonia/processes/ methanation.aspx.

17 M. Watanabe, H. Uchida, K. Ohkubo and H. Igarashi, Appl. Catal., B, 2003, 46, 595.

18 M. Kimura, T. Miyao, S. Komori, A. Chen, K. Higashiyama, H. Yamashita and M. Watanabe, Appl. Catal., A, 2010, 379, 182.

19 H. Harms, B. Hohlein, E. Jorn and A. Skov, Oil \& Gas Journal, 1980, 78(15), 120.

20 M. E. Dry, Appl. Catal., A, 2004, 276(1-2), 1-3.

21 M. A. Vannice, J. Catal., 1975, 37(3), 449-461.

22 T. Bligaard, J. K. Nørskov, S. Dahl, J. Matthiesen, C. H. Christensen and J. Sehested, J. Catal., 2004, 224(1), 206-217.

23 R. A. Dallabetta, A. G. Piken and M. Shelef, J. Catal., 1975, 40(2), 173-183.

24 T. Zubkov, G. A. Morgan and J. T. Yates, Chem. Phys. Lett., 2002, 362(3-4), 181-184.

25 T. Zubkov, G. A. Morgan, J. T. Yates, O. Kuhlert, M. Lisowski, R. Schillinger, D. Fick and H. J. Jansch, Surf. Sci., 2003, 526(1-2), $57-71$.

26 S. B. Vendelbo, M. Johansson, D. J. Mowbray, M. P. Andersson, F. Abild-Pedersen, J. H. Nielsen, J. K. Nørskov and I. Chorkendorff, Top. Catal., 2010, 53(5-6), 357-364.

27 N. M. Gupta, V. S. Kamble and R. M. Iyer, J. Catal., 1981, 69(1), $111-120$.

28 F. Abild-Pedersen, O. Lytken, J. Engbæk, G. Nielsen, I. Chorkendorff and J. K. Nørskov, Surf. Sci., 2005, 590(2-3), $127-137$.

29 X. D. Xiao, Y. L. Xie, C. Jakobsen, H. Galloway, M. Salmeron and Y. R. Shen, Phys. Rev. Lett., 1995, 74(19), 3860-3863.

30 T. Muller, D. Heuer, H. Pfnür and U. Kohler, Surf. Sci., 1996, 347(1-2), 80-96.

31 D. W. Goodman, Acc. Chem. Res., 1984, 17(5), 194-200.

32 R. D. Kelley and D. W. Goodman, The chemical physics of solid surfaces heterogeneous catalysis, Elsevier Scientific Publishing Company, NY, 1982, vol. 4, ch. 10, ISBN 0-444-41987-X.

33 J. A. Rodriguez and D. W. Goodman, Surf. Sci. Rep., 1991, 14(1-2), 1-107.

34 E. K. Vestergaard, R. T. Vang, J. Knudsen, T. M. Pedersen, T. An, E. Lægsgaard, I. Stensgaard, B. Hammer and F. Besenbacher, Phys. Rev. Lett., 2005, 95(12).

35 T. Gritsch, D. Coulman, R. J. Behm and G. Ertl, Phys. Rev. Lett., 1989, 63(10), 1086-1089.

36 B. J. Mcintyre, M. Salmeron and G. A. Somorjai, J. Vac. Sci. Technol., A, 1993, 11(4), 1964-1968.

37 B. L. M. Hendriksen, S. C. Bobaru and J. W. M. Frenken, Surf. Sci., 2004, 552(1-3), 229-242.

38 T. J. Udovic, R. D. Kelley and T. E. Madey, Surf. Sci., 1985, 150(1), L71-L76. 
39 J. Engbæk, O. Lytken, J. H. Nielsen and I. Chorkendorff, Surf. Sci., 2008, 602(3), 733-743.

40 H. Pfnur, P. Feulner and D. Menzel, J. Chem. Phys., 1983, 79(9), 4613-4623.

41 Y. Tison, K. Nielsen, D. J. Mowbray, L. Bech, and J. H. Nielsen, in preparation.

42 M. Johansson, J. H. Jørgensen and I. Chorkendorff, Rev. Sci. Instrum., 2004, 75(6), 2082-2093.
43 A. Johansson, T. Johannessen, J. H. Jørgensen and I. Chorkendorff, Appl. Surf. Sci., 2006, 252(10), 3673-3685.

44 R. C. Egeberg and I. Chorkendorff, Catal. Lett., 2001, 77(4), 207-213.

45 M. Johansson, I. Lundstrøm and L. G. Ekedahl, Rev. Sci. Instrum., 2000, 71(9), 3513-3521.

46 S. R. Kelemen and T. E. Fischer, Surf. Sci., 1979, 87(1), 53-68.

47 I. G. Bajusz and J. G. Goodwin, J. Catal., 1997, 169(1), 157-165.

48 C. S. Kellner and A. T. Bell, J. Catal., 1981, 70(2), 418-432. 